\title{
Three-Dimensional Hydrazone-Functionalized Covalent Organic Frameworks as pH-Triggered Rotary Switches
}

\author{
Wenjuan Zhao, Chengyang Yu, Fengqian Chen, Xinyu Guan, Hui Li, Bin Tang*, Valentin Valtchev, \\ Yushan Yan, Shilun Qiu, and Qianrong Fang*
}

\begin{abstract}
The property expansion of three-dimensional (3D) functionalized covalent organic frameworks (COFs) is important for developing their potential applications. Herein, we report the first case of $3 D$ hydrazone-decorated COFs as pH-triggered molecular switches, and explore their application in the stimuli-responsive drug delivery system. These functionalized COFs with hydrazone groups on the channel walls were obtained via a multi-component bottom-up synthesis strategy. They exhibit a reversible E/Z isomerization at various $p H$ values, confirmed by $U V$-vis absorption spectroscopy and proton conduction. Remarkably, after loading cytarabine (Ara-C) as a model drug molecule, these pH-responsive COFs showed an excellent and intelligent sustained-release effect with an almost 4fold increase in the Ara-C release at $\mathrm{pH}=4.8$ than at $\mathrm{pH}=7.4$, which will effectively improve drug-targeting and reduce drug side effects. Thus, these results open a way toward designing $3 D$ stimuliresponsive functionalized COF materials and promote their potential application as drug carriers in the field of disease treatment.
\end{abstract}

Covalent organic frameworks (COFs) are a new class of crystalline porous polymers that allow crystallographically precise integration of building blocks into periodic structures.$^{[1]}$ The potential applications of $\mathrm{COFs}$ are in various fields, including gas adsorption and separation, ${ }^{[2]}$ organic electronics, ${ }^{[3]}$ heterogeneous catalysis, ${ }^{[4]}$ and others. ${ }^{[5]}$ At present, most of the discovered COFs are still twodimensional (2D) frameworks with eclipsed stacking structures because of their simpler synthesis and easier functionalization.

[*] W. Zhao, C. Yu, F. Chen, X. Guan, Dr. H. Li, Prof. S. Qiu, Prof. Q. Fang

State Key Laboratory of Inorganic Synthesis and Preparative Chemistry, Jilin University,

Changchun 130012, China

E-mail: qrfang@jlu.edu.cn

Dr. B. Tang

Deakin University, Institute for Frontier Materials,

Geelong, Victoria 3216, Australia

E-mail: bin.tang@deakin.edu.au

Prof. V. Valtchev

Qingdao Institute of Bioenergy and Bioprocess

Technology, Chinese Academy of Sciences, Qingdao,

Shandong 266101, China

Prof. V. Valtchev

Normandie Univ, ENSICAEN, UNICAEN, CNRS,

Laboratoire Catalyse et Spectrochimie, 14050

Caen, France

Prof. Y. Yan

Department of Chemical and Biomolecular Engineering, Center for Catalytic Science and Technology,

University of Delaware,Newark, DE 19716, USA

Supporting information for this article is available on the WWW under http://www.angewandte.org or from the author. a)

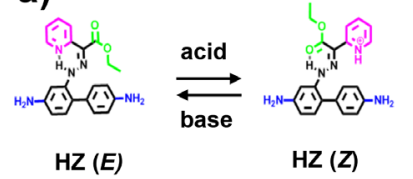

b)

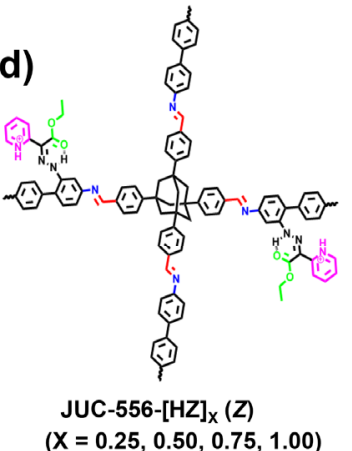

c)

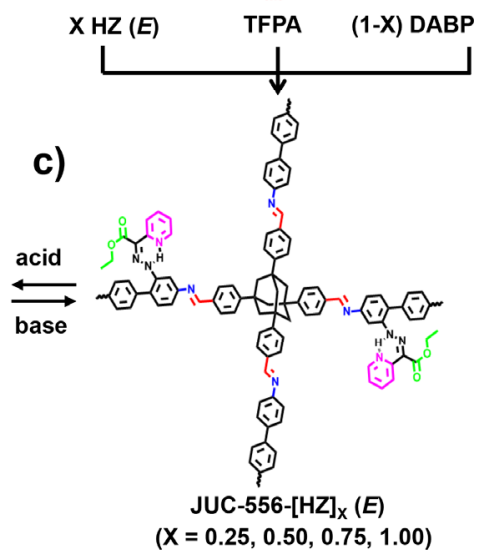

e)

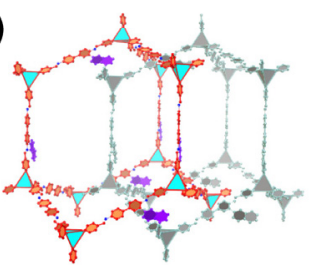

f)

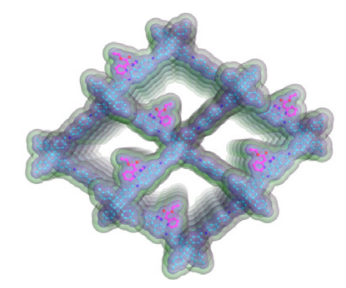

Scheme 1. Schematic representation of the strategy for preparing JUC-556-[HZ]x with $E / Z$ isomerization. (a) Acid/base controlled $E / Z$ isomerization of free $\mathrm{HZ}$ molecule. (b) Molecular structures of TFPA as a tetrahedral building unit as well as $\mathrm{HZ}(E)$ and DABP as linear linkers. (c) JUC-556-[HZ $]_{\mathrm{X}}(E)$ constructed by the condensation reaction of TFPA with $\mathrm{HZ}(E)$ and DABP $(\mathrm{X}=0.25$, $0.5,0.75$, and 1.00). (d) Acid/base controlled $E / Z$ isomerization in JUC-556-[HZ]X. (e) 2-fold interpenetrated dia network in JUC-556$[\mathrm{HZ}]_{\mathrm{X}}(E)$. (f) Structural representation of JUC-556-[HZ]X $(E)$.

Compared with 2D analogues, three-dimensional (3D) functionalized COFs have recently attracted more and more attention due to their unique pore structures and higher specific surface areas. ${ }^{[6]}$ For example, we have acquired a series of 3D functionalized $\mathrm{COFs},{ }^{[7]} \mathrm{e} . \mathrm{g}$., $3 \mathrm{D}$ tetrathiafulvalene-based $\mathrm{COFs}$ for tunable electrical conductivity, ${ }^{[7 \mathrm{a}]} 3 \mathrm{D}$ carboxy-functionalized $\mathrm{COF}$ for selective ion adsorption, ${ }^{[7 b]}$ and 3D Salphen-based COFs as catalytic antioxidants. ${ }^{[7 c]}$ Despite the aforementioned efforts in the in-situ synthesis and post-synthesis modifications, the functionalization of 3D COFs still remains largely undeveloped up to now, and especially 3D architectures with stimuli-responsive functions have been barely reported.

It is well-known that hydrazone and its derivatives are significant synthons for numerous transformations, and their $\mathrm{C}=\mathrm{N}$ groups can 
undergo efficiently reversible structures between $E$ and $Z$ configurations in the presence of acid or base (Scheme 1a). ${ }^{[8]}$ Consequently, $\mathrm{pH}$-triggered hydrazone-based switches are widely incorporated into various materials, ${ }^{[9]}$ including supramolecular systems, liquid crystals, and polymer gels to achieve such unique properties. Recently, some 2D hydrazone-based COFs have been reported, ${ }^{[10]}$ such as COF-42 ${ }^{[10 \mathrm{a}]}$ and TFPPy-DETHz-COF, ${ }^{[10 \mathrm{~b}]}$ in which hydrazone building units are used as the backbone of frameworks. Thus, their applications as stimuli-responsive materials are greatly hindered due to the restricted rotation. In principle, the combination of building blocks with hydrazone as side chains and the interconnected channels of 3D COFs will be greatly beneficial for the development of stimuli-responsive materials. However, no hydrazone has been integrated into 3D COFs because of the difficulties of crystallization and structural determination.

Herein, we report the synthesis of 3D hydrazone-equipped COFs and study their application as pH-triggered rotary switches in the stimuli-controlled release of the drug molecule. Different from previous reports, the hydrazone groups, in this case, were decorated on the channel walls of COFs using a multi-component bottom-up synthesis strategy. The obtained structures showed well-behaved invertibility of $E / Z$ isomerization at different $\mathrm{pH}$ values, which was confirmed by proton conduction and UV-vis absorption analysis. More importantly, after loading drug molecule (cytarabine, Ara-C), these stimuli-responsive COFs demonstrated exceptional release effects for Ara-C with an almost 4-fold amplification at $\mathrm{pH}=4.8$ than at $\mathrm{pH}=7.4$. This is the first example of $3 \mathrm{D}$ hydrazone-functionalized $\mathrm{COF}$ and their application for $\mathrm{pH}$-responsive drug delivery to the best of our knowledge.
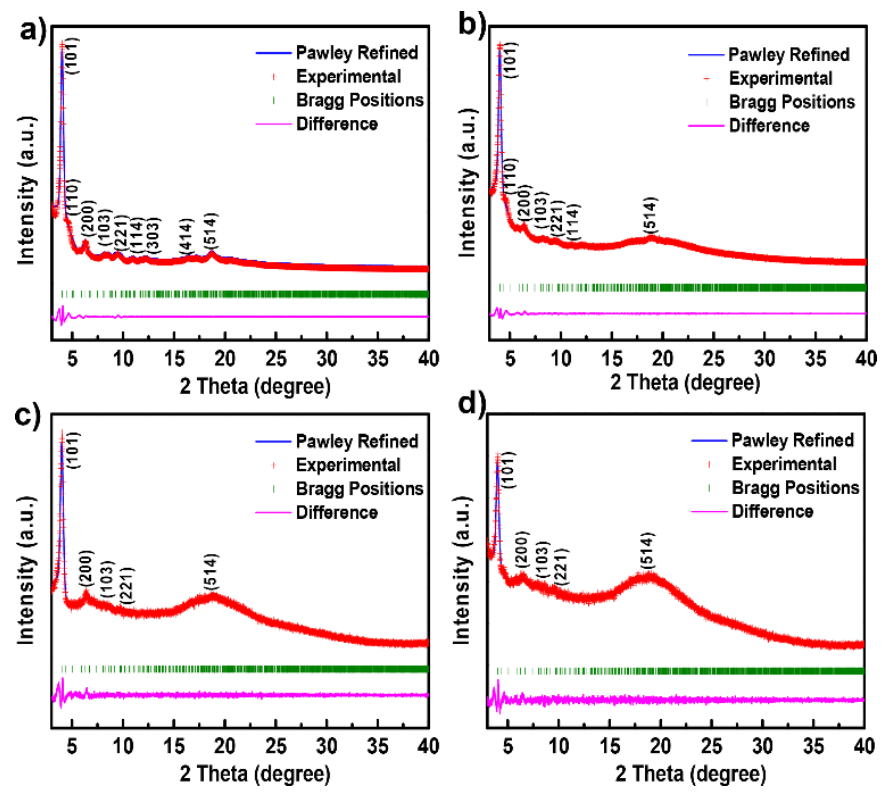

Figure 1. PXRD patterns of JUC-556-[HZ] $]_{0.25}(E)$ (a), JUC-556$[\mathrm{HZ}]_{0.50}(E)(\mathrm{b}), \mathrm{JUC}-556-[\mathrm{HZ}]_{0.75}(E)(\mathrm{c})$, and JUC-556-[HZ] $]_{1.00}(E)$ (d).

Our strategy for constructing 3D stimuli-responsive COFs is based on a hydrazone derivative with $E / Z$ interconversion, (E)-ethyl2-(2-(4,4'-diaminobiphenyl)hydrazono)-2-(pyridin-2-yl) acetate (HZ $(E)$, Scheme 1a). To maximize the functionalization of materials while maintaining their crystallinity and porosity, we have employed a multi-component condensation system to synthesize a series of 3D COFs. As shown in Scheme 1b, 4,4'-diaminobiphenyl (DABP) and $\mathrm{HZ}(E)$ were chosen as linear linkers, while 1,3,5,7-tetrakis(4formylphenyl)adamantane (TFPA) was designed as an ideal tetrahedral building unit. The condensation of TFPA with $\mathrm{HZ}(E)$ and DABP produced 3D COFs with different amounts of $\mathrm{HZ}(E)$, JUC$556-[\mathrm{HZ}] \mathrm{X}(E)$, where $\mathrm{X}$ is the proportion of $\mathrm{HZ}(E)(\mathrm{X}=[\mathrm{HZ}$ $(E)] /([\mathrm{DABP}]+[\mathrm{HZ}(E)])) ; \mathrm{X}=0.25,0.50,0.75$ or 1.00 ; Scheme $1 \mathrm{c})$. Similar to $\mathrm{HZ}(E)$ building unit, JUC-556-[HZ $]_{\mathrm{X}}(E)$ also displayed the excellent acid/base controlled $E / Z$ isomerization (Scheme $1 \mathrm{~d}$ ). Composed from linear and tetrahedral building blocks and subjected to the large steric effect of $\mathrm{HZ}(E)$ groups, these COFs are expected to exhibit a 2-fold interpenetrated diamondoid (dia) network (Scheme $1 \mathrm{e}){ }^{[11]}$

Typically, JUC-556-[HZ]x (E) were synthesized by suspending DABP and/or HZ $(E)$ with TFPA in the mixed solvent of dioxane and mesitylene in the presence of acetic acid followed by heating at $120{ }^{\circ} \mathrm{C}$ for 3 days. These condensation reactions exhibited similar isolated yields $(\sim 80 \%)$, indicating that the reactivities of DABP and $\mathrm{HZ}(E)$ were similar (Supporting Information, Section 1). The structural characterization of JUC-556-[HZ]X $(E)$ was executed combining complementary methods. Scanning electron microscopy (SEM) indicated that JUC-556-[HZ]x (E) showed rod-shaped crystals (Figures S1-4). In Fourier transform infrared (FTIR) spectra of JUC$556-[\mathrm{HZ}]_{\mathrm{X}}(E)$, the peaks assigned to $\mathrm{C}=\mathrm{N}$ stretching vibration appeared at about $1615 \mathrm{~cm}^{-1}$, demonstrating the formation of imine linkage (Figures S5-8). The solid-state ${ }^{13} \mathrm{C}$ cross-polarization magicangle-spinning (CP/MAS) NMR spectra further confirmed the presence of imine linkage in the light of the distinguishing $\mathrm{C}=\mathrm{N}$ signals at about $152 \mathrm{ppm}$ for JUC-556-[HZ]x (E) (Figures S9-12). According to the thermogravimetric analysis (TGA), JUC-556-[HZ]X (E) began to lose weight at $250{ }^{\circ} \mathrm{C}$ due to the decomposition of the $\mathrm{HZ}(E)$ monomer while the overall skeleton was stable up to about $500{ }^{\circ} \mathrm{C}$ in the nitrogen atmosphere (Figures S13-17). Furthermore, the crystalline structures of JUC-556-[HZ]x $(E)$ could be maintained in a variety of organic solvents and aqueous solutions with a series of $\mathrm{pH}$ values (Figures S18-21), verifying their remarkable stability.

The crystalline structures of JUC-556-[HZ]X $(E)$ were revealed by powder X-ray diffraction (PXRD) analysis (Figure 1). Herein we took JUC-556-[HZ] $]_{0.25}(E)$ as an example to analyze their structures (Figure 1a). The unit cell parameters of JUC-556-[HZ] $]_{0.25}(E)$ were resolved by the PXRD pattern in conjunction with structural simulation. After a geometrical energy minimization by using the Materials Studio software package ${ }^{[12]}$ based on a 2-fold interpenetrated dia net and disordered $\mathrm{HZ}(E),{ }^{[13]}$ the unit cell parameters of JUC-556-[HZ] $0.25(E)$ were obtained ( $a=b=28.2591$ $\AA, c=34.7253 \AA$ and $\alpha=\beta=\gamma=90^{\circ}$ ). The simulated PXRD pattern was in good agreement with the experimental one (Figure 1a). Furthermore, the full profile pattern matching (Pawley) refinement was performed from the experimental PXRD pattern. The strong PXRD peaks at 4.03, 5.11, 6.25, 8.27, 9.20, 11.10, 12.18, 16.42, and $18.98^{\circ} 2 \theta$ can be assigned to the (101), (110), (200), (103), (221), (114), (303), (414), and (514) Bragg peaks of a tetragonal space group $P 4_{2} /$ n (No. 86). The refinement results revealed that unit cell parameters were nearly equivalent to the predicted ones with excellent agreement factors $(a=b=28.3520 \AA, c=34.8160 \AA, \alpha=\beta$ $=\gamma=90^{\circ}, \omega R \mathrm{p}=5.77 \%$, and $R \mathrm{p}=4.29 \%$ ). In addition, we also examined alternative structures, such as non-interepenetrated dia network. However, there were significant differences between the 
simulated and experimental PXRDs (Figures S22-24). As the HZ (E) content increases, the crystallinity of JUC-556-[HZ $]_{\mathrm{X}}(E)$ decreases slightly due to disordered $\mathrm{HZ}(E)$ units on the channel walls; however, these materials also exhibit similar diffraction patterns, indicating that they have the same structures (Figure 1b-d). Based on the above results, it is proposed that JUC-556-[HZ]X $(E)$ have the expected architectures with 2 -fold interpenetrated dia nets, and microporous cavities with a diameter of about $1.70 \mathrm{~nm}$ (Scheme 1f).
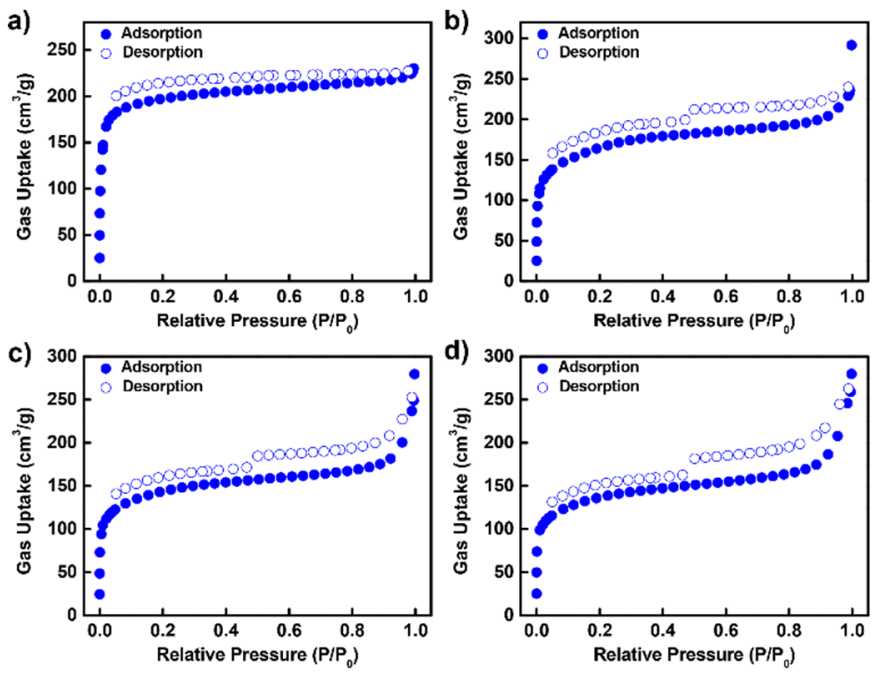

Figure 2. $\mathrm{N}_{2}$ adsorption-desorption isotherms for JUC-556-[HZ $]_{0.25}$ $(E)(\mathrm{a}), \mathrm{JUC}-556-[\mathrm{HZ}]_{0.50}(E)(\mathrm{b}), \mathrm{JUC}-556-[\mathrm{HZ}]_{0.75}(E)$ (c), and JUC$556-[\mathrm{HZ}]_{1.00}(E)(\mathrm{d})$ at $77 \mathrm{~K}$.

The porosity and specific surface areas of JUC-556-[HZ]x $(E)$ were analyzed by nitrogen gas adsorption measurements at $77 \mathrm{~K}$ (Figure 2). A sharp increase in gas uptake at low pressure (below 0.1 $\left.\mathrm{P} / \mathrm{P}_{0}\right)$ demonstrated the microporous nature of JUC-556-[HZ]x $(E)$. An inclination of the isotherm and slight desorption hysteresis was observed, implying the presence of textural mesopores caused by the agglomeration of COF crystals. ${ }^{[7 e]}$ Their surface areas exhibited a decreasing tendency with the increase of $\mathrm{HZ}(E)$ content. The Brunauer-Emmett-Teller (BET) surface areas were $634 \mathrm{~m}^{2} / \mathrm{g}$ for JUC$556-[\mathrm{HZ}]_{0.25}(E), 527 \mathrm{~m}^{2} / \mathrm{g}$ for JUC-556-[HZ] $]_{0.50}(E), 467 \mathrm{~m}^{2} / \mathrm{g}$ for JUC-556-[HZ] $0.75(E)$ and $430 \mathrm{~m}^{2} / \mathrm{g}$ for JUC-556-[HZ]1.00 $(E)$, respectively (Figures S25-28). Based on nonlocal density functional theory (NLDFT), JUC-556-[HZ]X $(E)$ showed similar microporous diameters of 1.62-1.80 nm (Figures S29-32), which were in good agreement with the pore sizes predicted from their crystal structures $(1.70 \mathrm{~nm})$.

Inspired by the abundant presence of $\mathrm{HZ}$ dangling groups, we studied the pH-triggered rotary switching effect of JUC-556-[HZ]x under different $\mathrm{pH}$ conditions (Figures 3 and S33-61). The acid/base induced $E / Z$ isomerization of dissociative $\mathrm{HZ}$ units was first inspected by UV-vis absorption spectroscopy, and the results clearly showed that the $\mathrm{pH}$-triggered switching process was reversible (Figures S3343). Similar to free HZ, JUC-556-[HZ]x also exhibited good pHresponsive switching behaviors. The color of the as-synthesized JUC$556-[\mathrm{HZ}]_{\mathrm{X}}(E)$ evolved gradually from yellow to red upon acid treatment, due to the sensitive $E / Z$ isomerization to various $\mathrm{pH}$ values (Figures $3 \mathrm{e}$ and S44). Furthermore, the evolution of UV-vis absorption spectra verified the $E / Z$ configurational changes. JUC$556-[\mathrm{HZ}]_{0.50}(E)$ was selected as an example to illustrate the acid-base isomerization. When JUC-556-[HZ] $]_{0.50}(E)$ was titrated with trifluoroacetic acid (TFA, Figures 3a and S45), the intensity of the absorption band at $283 \mathrm{~nm}$ decreased whereas that at $249 \mathrm{~nm}$ increased as the amount of the added acid increased. The spectral conversion clearly indicated that the HZ groups of the JUC-556$[\mathrm{HZ}]_{0.50}(E)$ underwent the configurational changes from $E$ to $Z$. Upon the addition of triethylamine $\left(\mathrm{Et}_{3} \mathrm{~N}\right)$ to the sample of JUC-556$[\mathrm{HZ}]_{0.50}(\mathrm{Z})$ (Figure $3 \mathrm{~b}$ and S46), the absorption band of $283 \mathrm{~nm}$ restored, accompanying with the declining of the absorption band at $249 \mathrm{~nm}$. JUC-556-[HZ] $]_{0.50}(E)$ also showed good pH-triggered switching processes upon the addition of $\mathrm{TFA} / \mathrm{Et}_{3} \mathrm{~N}$ with low concentrations and remarkable reversibility (Figure $3 \mathrm{c}$ and $3 \mathrm{~d}$ ). It should be noted that the transformation of colors and UV-vis absorption spectra of JUC-556-[HZ]X were slightly different from those of free HZ, which could be caused by steric hindrance and confinement effects of COF channels as well as potential inductive effects of atoms around $\mathrm{HZ}$ units. ${ }^{[8 \mathrm{a}]}$ Furthermore, the spectral conversion of JUC-556-[HZ] $]_{0.50}(E)$ occurred with other acids, such as $\mathrm{HCl}$ (Figures $3 \mathrm{f}$ and $\mathrm{S} 47$ ). As for other JUC-556-[HZ $]_{\mathrm{X}}(E)$, similar acid/base dependent changes of UV-vis absorption spectra were observed, and their $E / Z$ configurations were also reversible under acidic/basic conditions (Figures S48-61).
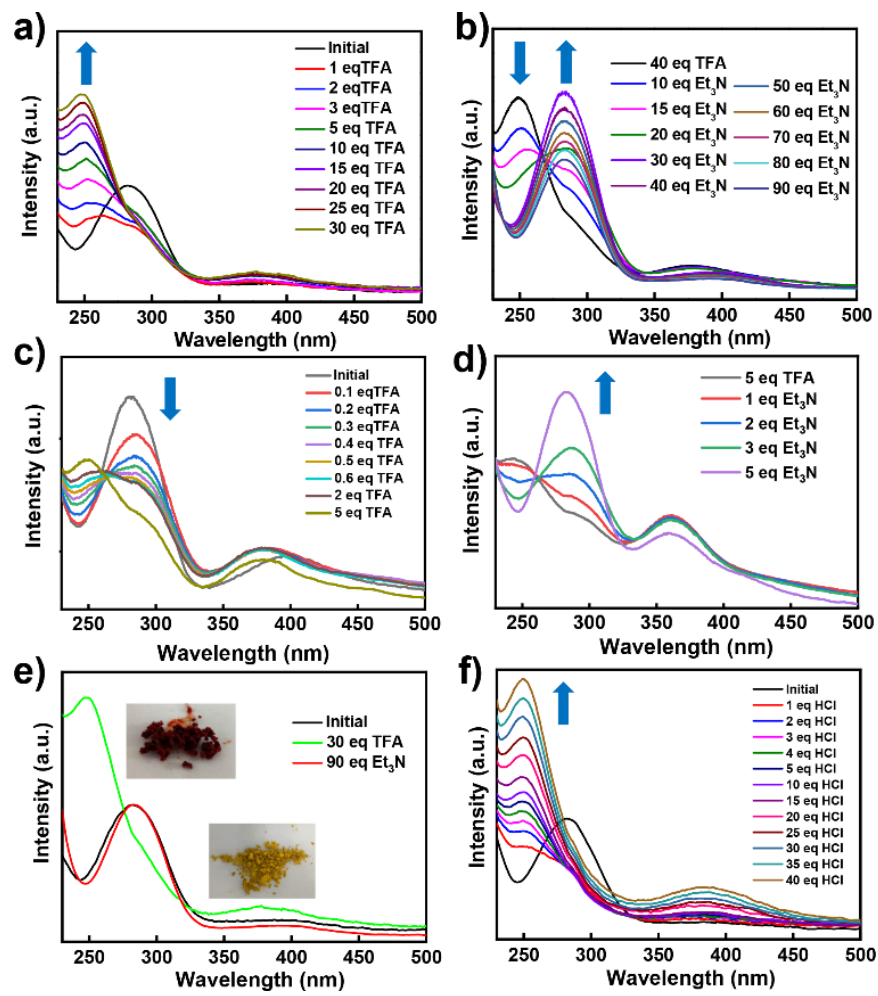

Figure 3. (a) Absorption spectra of JUC-556-[HZ $]_{0.50}$ (E) upon protonation in TFA solution with increasing concentrations. (b) Absorption spectra of JUC-556-[HZ] $]_{0.50}(Z)$ upon deprotonation in $\mathrm{Et}_{3} \mathrm{~N}$ solution with increasing concentrations. (c) Absorption spectra of JUC-556-[HZ] $]_{0.50}(E)$ upon protonation in TFA solution under low concentrations. (d) Absorption spectra of JUC-556-[HZ]0.50 (Z) upon deprotonation in $\mathrm{Et}_{3} \mathrm{~N}$ solution under low concentrations. (e) Reversible change of JUC-556-[HZ] $]_{0.50}(E)$ in the acid/base solution. Inset: the color change of JUC-556-[HZ] $]_{0.50}(E)$. (f) Absorption spectra of JUC-556-[HZ] $]_{0.50}(E)$ upon protonation in $\mathrm{HCl}$ solution with increasing concentrations. 
Furthermore, the $E / Z$ isomerization of JUC-556-[HZ]x was demonstrated by proton conduction at room temperature (Figures 4 and S62-65). As HZ (E) has rich nitrogen atoms, it can combine with $\mathrm{HCl}$ to form hydrogen bonds, ${ }^{[14]}$ and therefore the as-synthesized JUC-556-[HZ $]_{\mathrm{X}}(E)$ possessed the ability to accept protons. Typically, JUC-556-[HZ $]_{\mathrm{X}}(E)$ were compressed into cylindrical pellets with a diameter of $6.0 \mathrm{~mm}$ and a thickness of $1.0 \mathrm{~mm}$. The proton conductivities of the original JUC-556-[HZ]x $(E)$ were $2.73 \times 10^{-6} \mathrm{~S}$ $\mathrm{m}^{-1}$ for JUC-556-[HZ] $]_{0.25}(E), 3.70 \times 10^{-7} \mathrm{~S} \mathrm{~m}^{-1}$ for JUC-556-[HZ] 0.50 (E), $1.32 \times 10^{-6} \mathrm{~S} \mathrm{~m}^{-1}$ for JUC-556-[HZ] $0.75(E)$, and $3.91 \times 10^{-7} \mathrm{~S} \mathrm{~m}^{-1}$ for JUC-556-[HZ]1.00 (E), respectively. Remarkably, upon protonation with $\mathrm{HCl}$ vapor, the proton conductivities of activated JUC-556-[HZ]x $(Z)$ increased up to $4.41 \times 10^{-4} \mathrm{~S} \mathrm{~m}^{-1}$ for JUC-556$[\mathrm{HZ}]_{0.25}(Z), 7.04 \times 10^{-4} \mathrm{~S} \mathrm{~m}^{-1}$ for JUC-556-[HZ] $]_{0.50}(Z), 2.17 \times 10^{-4} \mathrm{~S}$ $\mathrm{m}^{-1}$ for JUC-556-[HZ] $]_{0.75}(Z)$, and $3.30 \times 10^{-4} \mathrm{~S} \mathrm{~m}^{-1}$ for JUC-556$[\mathrm{HZ}]_{1.00}(\mathrm{Z})$, which are 162-fold, 1903-fold, 164-fold, and 844-fold improvement than those of the original ones, respectively. In addition, the proton conductivity dropped when the JUC-556-[HZ]x $(Z)$ were treated with $\mathrm{Et}_{3} \mathrm{~N}$ vapor, indicating that the $\mathrm{pH}$ switching process was reversible.
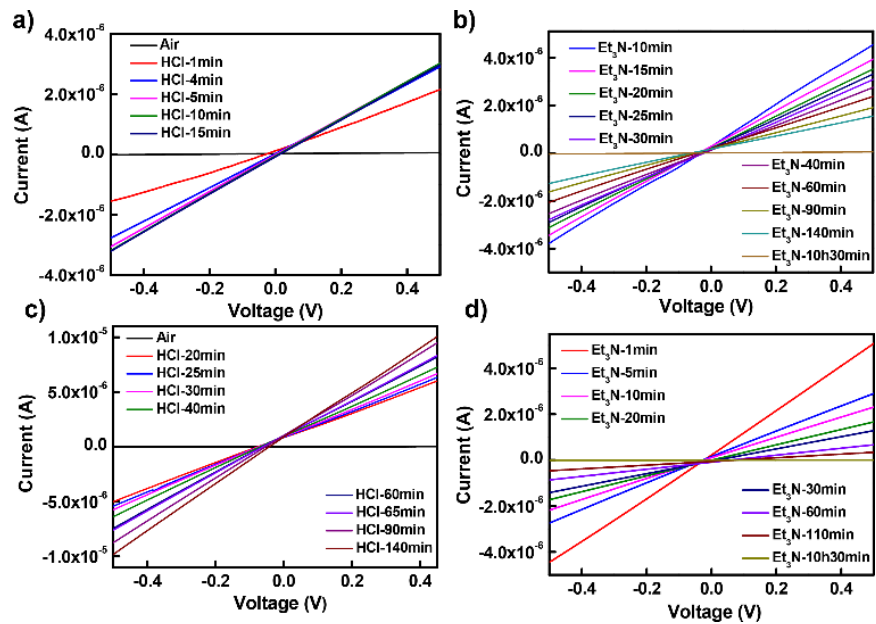

Figure 4. (a) Proton conductivity of activated JUC-556-[HZ $]_{0.25}(E)$ in contact with $\mathrm{HCl}$ vapor. (b) Proton conductivity of JUC-556$[\mathrm{HZ}]_{0.25}(Z)$ in contact with $\mathrm{Et}_{3} \mathrm{~N}$ vapor. (c) Proton conductivity of activated JUC-556-[HZ $]_{0.50}(E)$ in contact with $\mathrm{HCl}$ vapor. (d) Proton conductivity of JUC-556-[HZ] $]_{0.50}(Z)$ in contact with $\mathrm{Et}_{3} \mathrm{~N}$ vapor.

Given the high porosity and stability of JUC-556-[HZ]x (E) as well as favorable $\mathrm{pH}$-responsive $\mathrm{HZ}$ units, we explored their potential application in the stimuli-responsive drug delivery system. Ara-C with a molecular size of about $0.9 \mathrm{~nm}$ was chosen as a model molecule because it is a traditional drug for cancer therapy, especially for pancreatic cancer, acute myelogenous leukemia and chronic lymphoma. ${ }^{[15]}$ Typically, the JUC-556-[HZ]x $(E)$ were immersed in Ara-C aqueous solution for $9 \mathrm{~h}$ under stirring at $200 \mathrm{rpm}$, and Ara-Cloaded JUC-556-[HZ]x $(E)$ were confirmed by UV-vis absorption spectra (Figure S66). Then, the mixtures were filtered and washed. The resultant PXRD peaks were coincided with those of starting materials, confirming the structural integrity after loading Ara-C (Figures S67-70). Each Ara-C-loaded JUC-556-[HZ]x (E) was transferred to two ampoules containing $5.0 \mathrm{~mL}$ releasing medium separately, and the maximium UV-vis absorption intensity at $272 \mathrm{~nm}$ was chosen to indicate the drug concentration during the drugreleasing process. It is known that the cancer tissues generally exist in acidic extracellular environments with $\mathrm{pH}=4$ to 6 . Therefore, we chose acetic acid buffer with $\mathrm{pH}=4.8$ as simulated cancer fluid to release the drug. The $\mathrm{pH}$ value in the normal physiological environment is almost neutral, and so a phosphate buffer $(\mathrm{pH}=7.4)$ was used to simulate normal body fluid. ${ }^{[16]}$ All JUC-556-[HZ]X showed smart drug sustained-release effects in two buffer solutions (Figures 5b and S71-73). Among them, JUC-556-[HZ]0.50 displayed the best performance in the release of drug, and the release rate reached $74.56 \%$ in the $\mathrm{pH}=4.8$ buffer within $72 \mathrm{~h}$ ( $Z$ isomerization), but only $18.59 \%$ in the $\mathrm{pH}=7.4$ buffer ( $E$ isomerization, Figure $5 \mathrm{~b}$ ). The release rate of drug molecule under acidic condition was nearly 4-fold higher than that under neutral condition, greatly improving the drug targeting delivery and reducing its side effects. Furthermore, JUC-556-[HZ] $]_{0.50}$ with $E$ or $Z$ isomerization exhibited reproducible identical effect for the capture and release of Ara-C even after 5 cycles (Figure 5c).

As for the drug release mechanism, it is suggested that the $\mathrm{HZ}$ unit as a $\mathrm{pH}$-responsive rotary switch in JUC-556-[HZ]x plays a key role, which can form a variety of hydrogen bonds with drug molecules. ${ }^{[17]}$ When the solution is neutral or basic, the hydrogen bonds between Ara-C and $\mathrm{HZ}(E)$ units promote the loading of AraC onto JUC-556-[HZ]x $(E)$. Meanwhile, the channels of JUC-556$[\mathrm{HZ}] \mathrm{x}(E)$ provide the platforms for encapsulating Ara-C. On the contrary, when the surrounding is acidic, JUC-556-[HZ]X $(E)$ transforms to $Z$-type configuration through the protonation of pyridine subunits, and simultaneously Ara-C was also protonated. The repulsive effect between JUC-556-[HZ $]_{\mathrm{X}}(Z)$ and Ara-C can drive the high-efficiency release of Ara-C at the acidic condition (Figure 5a).

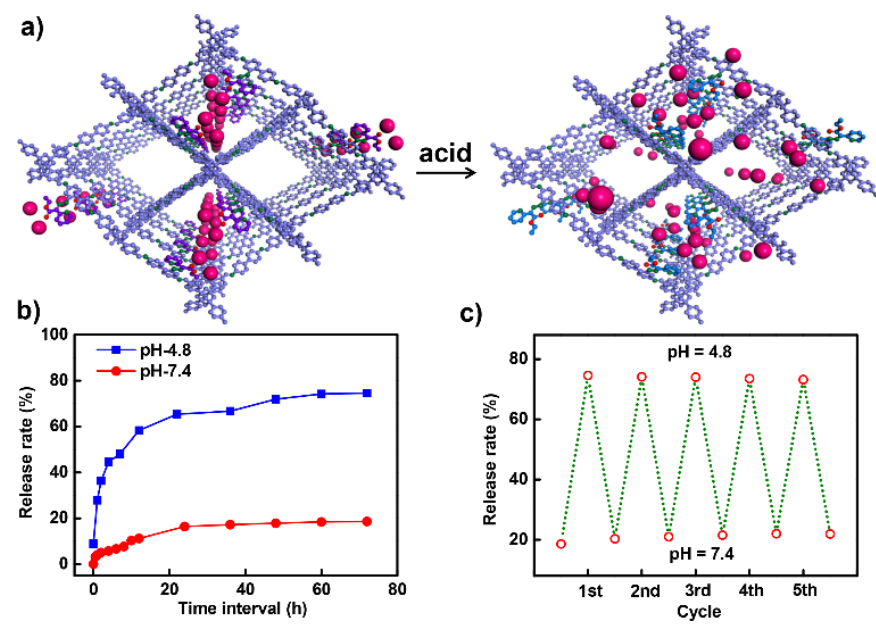

Figure 5. (a) Schematic representation of the release of Ara-C from the channels of JUC-556-[HZ]x in acidic solution. Drug release profiles (b) and reversibility (c) of Ara-C-loaded JUC-556-[HZ] $]_{0.50}$ in simulated cancer fluid $(\mathrm{pH}=4.8$ buffer solution) and in simulated normal body fluid ( $\mathrm{pH}=7.4$ buffer solution).

In conclusion, we have synthesized a series of novel 3D hydrazone-decorated COFs, JUC-556-[HZ]x $(E)$, as pH-triggered rotary switches via the bottom-up multi-component approach. JUC556-[HZ]x $(E)$ showed high crystallinity, good chemical stability, and reversible $E / Z$ isomerization at various $\mathrm{pH}$ values, verified by $U V$-vis absorption spectroscopy and proton conduction. Furthermore, these functionalized COF materials were applied to intelligent $\mathrm{pH}$ - 
responsive Ara-C delivery and exhibited a nearly 4-fold increase in the drug release at $\mathrm{pH}=4.8$ than at $\mathrm{pH}=7.4$, which will effectively improve drug-targeting and reduce drug side effects. This study thus develops the design and synthesis of 3D stimuli-responsive COFs, and promotes the potential application of COF materials for disease theranostics.

Further detailed experimental procedures and characterization are described in the Supporting Information.

\section{Acknowledgments}

This work was supported by the National Natural Science Foundation of China (22025504, 21621001, and 21390394), "111" project (BP0719036 and B17020), China Postdoctoral Science Foundation (2020TQ0118 and 2020M681034) and the program for JLU Science and Technology Innovative Research Team. V.V., Q.F. and S.Q. acknowledge the collaboration in the framework of China-French joint laboratory "Zeolites".

\section{Conflict of interest}

The authors declare no conflict of interest.

Received: ((will be filled in by the editorial staff))

Published online on ((will be filled in by the editorial staff))

Keywords: covalent organic frameworks $\cdot$ functionalization stimuli-responsive $\cdot$ drug release

[1] a) A. P. Côté, A. I. Benin, N. W. Ockwig, M. O'Keeffe, A. J. Matzger, O. M. Yaghi, Science 2005, 310, 1166-1170; b) J. W. Colson, W. R. Dichtel, Nat. Chem. 2013, 5, 453-465; c) S. Y. Ding, W. Wang, Chem. Soc. Rev. 2013, 42, 548-568; d) J. L. Segura, M. J. Mancheño, F. Zamora, Chem. Soc. Rev. 2016, 45, 5635-5671; e) M. S. Lohse, T. Bein, Adv. Funct. Mater. 2018, 28, 1705553; f) Y. Yusran, X. Y. Guan, H. Li, Q. R. Fang, S. L. Qiu, Nat. Sci. Rev. 2020, 7, 170-190; g) Z. F. Wang, S. N. Zhang, Y. Chen, Z. J. Zhang, S. Q. Ma, Chem. Soc. Rev. 2020, 49, 708735; h) X. Y. Guan, F. Q. Chen, Q. R. Fang, S. L. Qiu, Chem. Soc. Rev. 2020, 49, 1357-1384; i) K. Y. Geng, T. He, R. Y. Liu, S. Dalapati, K. T. Tan, Z. P. Li, S. S. Tao, Y. F. Gong, Q. H. Jiang, D. L. Jiang, Chem. Rev. 2020, 120, 8814-8933.

[2] a) S. S. Han, H. Furukawa, O. M. Yaghi, W. A. Goddard III, J. Am. Chem. Soc. 2008, 130, 11580-11581; b) P. Kuhn, M. Antonietti, A. Thomas, Angew. Chem. Int. Ed. 2008, 47, 34503453; Angew. Chem. 2008, 120, 3499-3502; c) S. Wang, Q. Y. Wang, P. P. Shao, Y. Z. Han, X. Gao, L. Ma, S. Yuan, X. J. Ma, J. W. Zhou, X. Feng, B. Wang, J. Am. Chem. Soc. 2017, 139, 4258-4261; d) H. Furukawa, O. M. Yaghi, J. Am. Chem. Soc. 2009, 131, 8875-8883; e) Y. J. Wang, Y. Z. Liu, H. Li, X. Y. Guan, M. Xue, Y. S. Yan, V. Valtchev, S. L. Qiu, Q. R. Fang, J. Am. Chem. Soc. 2020, 142, 3736-3741; f) S. S. Yuan, X. Li, J. Y. Zhu, G. Zhang, P. Van Puyvelde, B. Van der Bruggen, Chem. Soc. Rev. 2019, 48, 2665-2681;

[3] a) S. Wan, J. Guo, J. Kim, H. Ihee, D. L. Jiang, Angew. Chem. Int. Ed. 2008, 47, 8826-8830; Angew. Chem. 2008, 120, 8958-
8962; b) E. L. Spitler, B. T. Koo, J. L. Novotney, J. W. Colson, F. J. Uribe-Romo, G. D. Gutierrez, P. Clancy, W. R. Dichtel, J. Am. Chem. Soc. 2011, 133, 19416-19421; c) X. S. Ding, J. Guo, X. Feng, Y. Honsho, J. D. Guo, S. Seki, P. Maitarad, A. Saeki, S. Nagase, D. L. Jiang, Angew. Chem. Int. Ed. 2011, 50, 12891293; Angew. Chem. 2011, 123, 1325-1329; d) G. H. V. Bertrand, V. K. Michaelis, T. C. Ong, R. G. Griffin, M. Dinca, Proc. Natl. Acad. Sci. USA 2013, 110, 4923-4928; e) M. Dogru, M. Handloser, F. Auras, T. Kunz, D. Medina, A. Hartschuh, P. Knochel, T. Bein, Angew. Chem. Int. Ed. 2013, 52, 2920-2924; Angew. Chem. 2013, 125, 2992-2996; f) Y. Du, H. Yang, J. M. Whiteley, S. Wan, Y. Jin, S. H. Lee, W. Zhang, Angew. Chem. Int. Ed. 2016, 55, 1737-1741; Angew. Chem. 2016, 128, 17691773; g) M. Calik, F. Auras, L. M. Salonen, K. Bader, I. Grill, M. Handloser, D. D. Medina, M. Dogru, F. Löbermann, D. Trauner, A. Hartschuh, T. Bein, J. Am. Chem. Soc. 2014, 136, 17802-17807; h) P. P. Shao, J. Li, F. Chen, L. Ma, Q. B. Li, M. X. Zhang, J. W. Zhou, A. X. Yin, X. Feng, B. Wang, Angew. Chem. Int. Ed. 2018, 57, 16501-16505; Angew. Chem. 2018, 130, 16654-16658.

[4] a) S. Y. Ding, J. Gao, Q. Wang, Y. Zhang, W. G. Song, C. Y. Su, W. Wang, J. Am. Chem. Soc. 2011, 133, 19816-19822; b) S. Lin, C. S. Diercks, Y. B. Zhang, N. Kornienko, E. M. Nichols, Y. Zhao, A. R. Paris, D. Kim, P. Yang, O. M. Yaghi, C. J. Chang, Science 2015, 349, 1208-1213; c) Q. R. Fang, S. Gu, J. Zheng, Z. B. Zhuang, S. L. Qiu, Y. S. Yan, Angew. Chem. Int. Ed. 2014, 53, 2878-2882; Angew. Chem. 2014, 126, 2922-2926; d) Y. W. Peng, Z. G. Hu, Y. J. Gao, D. Q. Yuan, Z. X. Kang, Y. H. Qian, N. Yan, D. Zhao, ChemSusChem 2015, 8, 3208-3212; e) V. S. Vyas, F. Haase, L. Stegbauer, G. Savasci, F. Podjaski, C. Ochsenfeld, B. V. Lotsch, Nat. Commun. 2015, 6, 8508; f) X. R. Wang, X. Han, J. Zhang, X. W. Wu, Y. Liu, Y. Cui, J. Am. Chem. Soc. 2016, 138, 12332-12335; g) Q. Sun, B. Aguila, J. Perman, N. Nguyen, S. Q. Ma, J. Am. Chem. Soc. 2016, 138, 1579015796; h) H. C. Ma, C. C. Zhao, G. J. Chen, Y. B. Dong, Nat. Commun. 2019, 10, 3368.

[5] a) C. R. DeBlase, K. E. Silberstein, T. Truong, H. D. Abruña, W. R. Dichtel, J. Am. Chem. Soc. 2013, 135, 16821-16824; b) C. J. Doonan, D. J. Tranchemontagne, T. G. Glover, J. R. Hunt, O. M. Yaghi, Nat. Chem. 2010, 2, 235-238; c) T. Y. Zhou, S. Q. Xu, Q. Wen, Z. F. Pang, X. Zhao, J. Am. Chem. Soc. 2014, 136, 1588515888; d) Q. R. Fang, Z. B. Zhuang, S. Gu, R. B. Kaspar, J. Zheng, J. H. Wang, S. L. Qiu, Y. S. Yan, Nat. Commun. 2014, 5, 4503; e) Q. Sun, B. Aguila, J. Perman, L. D. Earl, C. W. Abney, Y. Cheng, H. Wei, N. Nguyen, L. Wojtas, S. Q. Ma, J. Am. Chem. Soc. 2017, 139, 2786-2793; f) H. P. Ma, B. L. Liu, B. Li, L. M. Zhang, Y. G. Li, H. Q. Tan, H. Y. Zang, G. S. Zhu, J. Am. Chem. Soc. 2016, 138, 5897-5903; g) J. Roeser, D. Prill, M. J. Bojdys, P. Fayon, A. Trewin, A. N. Fitch, M. U. Schmidt, A. Thomas, Nat. Chem. 2017, 9, 977-982; h) X. Y. Guan, H. Li, Y. C. Ma, M. Xue, Q. R. Fang, Y. S. Yan, V. Valtchev, S. L. Qiu, Nat. Chem. 2019, 11, 587-594; i) H. Lyu, C. S. Diercks, C. H. Zhu, O. M. Yaghi, J. Am. Chem. Soc. 2019, 141, 6848-6852; j) Y. Z. Liu, Y. J. Wang, H. Li, X. Y. Guan, L. K. Zhu, M. Xue, Y. S. Yan, V. Valtchev, S. L. Qiu, Q. R. Fang, Chem. Sci. 2019, 10, 10815-10820; j) H, Wang, C. Qian, J. Liu, Y. F. Zeng, D. D. Wang, W. Q. Zhou, L. Gu, H. W. Wu, G. F. Liu, Y. L. Zhao, J. Am. Chem. Soc. 2020, 142, 4862-4871; k) Y. Yusran, H. Li, X. Y. Guan, D. H. Li, L. X. Tang, M. Xue, Z. B. Zhuang, Y. S. Yan, V. Valtchev, S. L. Qiu, Q. R. Fang, Adv. Mater. 2020, 32, 
1907289; 1) D. H. Li, C. Y. Li, L. J. Zhang, H. Li, L. K. Zhu, D. J. Yang, Q. R. Fang, S. L. Qiu, X. D. Yao, J. Am. Chem. Soc. 2020, 142, 8104-8108.

[6] a) H. M. El-Kaderi, J. R. Hunt, J. L. Mendoza-Cortes, A. P. Côté, R. E. Taylor, M. O'Keeffe, O. M. Yaghi, Science 2007, 316, 268272; b) Y. B. Zhang, J. Su, H. Furukawa, Y. F. Yun, F. Gandara, A. Duong, X. D. Zou, O. M. Yaghi, J. Am. Chem. Soc. 2013, 135, 16336-16339; c) D. Beaudoin, T. Maris, J. D. Wuest, Nat. Chem. 2013, 5, 830-834; d) G. Q. Lin, H. M. Ding, D. Q. Yuan, B. S. Wang, C. Wang, J. Am. Chem. Soc. 2016, 138, 3302-3305; e) Y. Z. Liu, Y. H. Ma, Y. B. Zhao, X. X. Sun, F. Gándara, H. Furukawa, Z. Liu, H. Y. Zhu, C. H. Zhu, K. Suenaga, P. Oleynikov, A. S. Alshammari, X. Zhang, O. Terasaki, O. M. Yaghi, Science 2016, 351, 365-369; f) L. A. Baldwin, J. W. Crowe, D. A. Pyles, P. L. McGrier, J. Am. Chem. Soc. 2016, 138, 15134-15137; g) Y. X. Ma, Z. J. Li, L. Wei, S. Y. Ding, Y. B. Zhang, W. Wang, J. Am. Chem. Soc. 2017, 139, 4995-4998; h) T. Q. Ma, E. A. Kapustin, S. X. Yin, L. Liang, Z. Y. Zhou, J. Niu, L. H. Li, Y. Y. Wang, J. Su, J. Li, X. G. Wang, W. D. Wang, W. Wang, J. L. Sun, O. M. Yaghi, Science 2018, 361, 48-52; i) Y. C. Ma, Y. J. Wang, H. Li, X. Y. Guan, B. J. Li, M. Xue, Y. S. Yan, V. Valtchev, S. L. Qiu, Q. R. Fang, Angew. Chem. Int. Ed. 2020, 59, 19633-19638; Angew. Chem. 2020, 132, 19744-19749.

[7] a) H. Li, J. H. Chang, S. S. Li, X. Y. Guan, D. H. Li, C. Y. Li, L. X. Tang, M. Xue, Y. S. Yan, V. Valtchev, S. L. Qiu, Q. R. Fang, J. Am. Chem. Soc. 2019, 141, 13324-13329; b) Q. Y. Lu, Y. C. Ma, H. Li, X. Y. Guan, Y. Yusran, M. Xue, Q. R. Fang, Y. S. Yan, S. L. Qiu, V. Valtchev, Angew. Chem. Int. Ed. 2018, 57, 60426048; Angew. Chem. 2018, 130, 6150-6156; c) S. C. Yan, X. Y. Guan, H. Li, D. H. Li, M. Xue, Y. S. Yan, V. Valtchev, S. L. Qiu, Q. R. Fang, J. Am. Chem. Soc. 2019, 141, 2920-2924; d) Z. L. Li, H. Li, X. Y. Guan, J. J. Tang, Y. Yusran, Z. Li, M. Xue, Q. R. Fang, Y. S. Yan, V. Valtchev, S. L. Qiu, J. Am. Chem. Soc. 2017, 139, 17771-17774; e) Q. R. Fang, J. H. Wang, S. Gu, R. B. Kaspar, Z. B. Zhuang, J. Zheng, H. X. Guo, S. L. Qiu, Y. S. Yan, J. Am. Chem. Soc. 2015, 137, 8352-8355; f) H. Li, Q. Y. Pan, Y. C. Ma, X. Y. Guan, M. Xue, Q. R. Fang, Y. S.Yan, V. Valtchev, S. L. Qiu, J. Am. Chem. Soc. 2016, 138, 14783-14788; g) X. Y. Guan, Y. C. Ma, H. Li, Y. Yusran, M. Xue, Q. R. Fang, Y. S. Yan, V. Valtchev, S. L. Qiu, J. Am. Chem. Soc. 2018, 140, 4494-4498; h) H. Li, J. H. Ding, X. Y. Guan, F. Q. Chen, C. Y. Li, L. K. Zhu, M. Xue, D. Q. Yuan, V. Valtchev, Y. S. Yan, S. L. Qiu, Q. R. Fang, J. Am. Chem. Soc. 2020, 142, 13334-13338.

[8] a) X. Su, I. Aprahamian, Chem. Soc. Rev. 2014, 43, 1963-1981; b) S. M. Landge, E. Tkatchouk, D. Benítez, D. A. Lanfranchi, M. Elhabiri, W. A. Goddard, I. Aprahamian, J. Am. Chem. Soc. 2011, 133, 9812-9823.

[9] a) S. Kassem, A. T. L. Lee, D. A. Leigh, A. Markevicius, J. Solà, Nat. Mater. 2016, 8, 138-143; b) S. M. Landge, I. Aprahamian, J. Am. Chem. Soc. 2009, 131, 18269-18271; c) B. Shao, M. Baroncini, H. Qian, L. Bussotti, M. D. Donato, A. Credi, I. Aprahamian, J. Am. Chem. Soc. 2018, 140, 12323-12327; d) X. Su, S.Voskian, R. P. Hughes, I. Aprahamian, Angew. Chem. Int. Ed. 2013, 52, 10734-10739; Angew. Chem. 2013, 125, 1093410939.

[10] a) F. J. Uribe-Romo, C. J. Doonan, H. Furukawa, K. Oisaki, O. M. Yaghi, J. Am. Chem. Soc. 2011, 133, 11478-11481; b) Z. Li, N. Huang, H. Ka, Y. Feng, S. Tao, Q Jiang, N. Yuki, I. Stephan, D. Jiang, J. Am. Chem. Soc. 2018, 140, 12374-12377; c) D. N. Bunck, W. R. Dichtel, J. Am. Chem. Soc. 2013, 135, 14952-
14955; d) L. Stegbauer, K. Schwinghammer, B. V. Lotsch, Chem. Sci. 2014, 5, 2789-2793; e) X. Li, Q. Gao, J. Wang, Nat. Commun. 2018, 9, 2335.

[11] C. Bonneau, O. Delgado-Friedrichs, M. O'Keeffe, O. M. Yaghi, Acta Crystallogr. Sect. A 2004, 60, 517-520.

[12] Materials Studio ver. 7.0; Accelrys Inc.; San Diego, CA.

[13] A. Nagai, Z. Q. Guo, X. Feng, S. B. Jing, X. Chen, X. S. Ding, D. L. Jiang, Nat. Commun. 2011, 2, 536.

[14] a) R. Kulkarni, Y. Noda, D. K. Barange, Y. S. Kochergin, P. Lyu, B. Balcarova, P. Nachtigall, M. J. Bojdys, Nat. Commun. 2019, 10, 3228; b) D. Chen, W. Chen, G. Xing, T. Zhang, L. Chen, Chem. Eur. J. 2020, 26, 8377-8381.

[15] N. Martinez, B. Drescher, H. Riehle, C. Cullmann, H. P. Vornlocher, A. Ganser, G. Heil, A. Nordheim, J. Krauter, O. Heidenreich, BMC Cancer 2004, 4, 44.

[16] J. H. Kim, Y. S. Kim, K. Park, S. Lee, H. Y. Nam, K. H. Min, H. G. Jo, J. H. Park, K. Choi, S. Y. Jeong, R.W. Park, I. S. Kim, K. Kim, I. C. Kwon, J. Control. Release 2008, 127, 41-49.

[17] a) J. Chen, B. Zhang, F. Xia, Y. Xie, S. Jiang, R. Su, W. $\mathrm{Wu}$, Nanoscale 2016, 8, 7127-7136; b) R. Ocampo-Pérez, M. Sánchez-Polo, J. Rivera-Utrilla, R. Leyva-Ramos, Chem. Eng. J. 2010, 165, 581-588; c) S. J. Sonawane, R. S. Kalhapure, T. Govender, Eur. J. Pharm. Sci. 2017, 99 , 45-65. 


\section{Covalent Organic Frameworks}

Wenjuan Zhao, Chengyang Yu, Fengqian Chen, Xinyu Guan, Hui Li, Bin Tang*, Valentin Valtchev, Yushan Yan, Shilun Qiu, and Qianrong Fang*

\section{Page - Page}

Three-Dimensional Hydrazone-

Functionalized Covalent Organic Frameworks as $\mathrm{pH}$-Triggered Rotary Switches
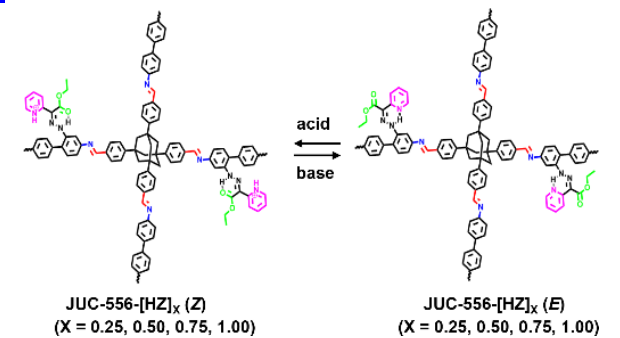

Here we report the first case of $3 \mathrm{D}$ hydrazone-decorated $\mathrm{COFs}$ as $\mathrm{pH}$-triggered molecular switches, and explore their application in the stimuli-responsive drug delivery system. 